\title{
Developmental Induction and Villus-Crypt Distribution of Retinol Esterifying Enzyme Activities in Chick Duodenum
}

\author{
Sanae Tajima, Kazuhito Suruga, Toshinao GodA* \\ and Sachiko TAKASE \\ School of Food and Nutritional Sciences, University of Shizuoka, \\ Shizuoka 422-8526, Japan
}

(Received July 26, 1999)

\begin{abstract}
Summary Retinol absorbed and generated from dietary $\beta$-carotene can be esterified by retinol esterifying enzyme(s) in intestinal absorptive cells. In this study, we observed the developmental changes and villus-crypt distribution of the activities of two retinol esterifying enzymes (lecithinretinol acyltransferase (LRAT); and acyl-CoA-retinol acyltransferase (ARAT) in chick duodenum) to seek the possibility that these enzymes play distinct roles in retinol absorption and metabolism. Intestinal LRAT activity was barely expressed in embryonic stages until $2-3 \mathrm{~d}$ before hatching, when its activity becomes detectable; thereafter it abruptly increased to the maximal level at the third day of the posthatch period. In contrast, ARAT activity was present in the duodenum at the earliest stage examined, the 15th day of embryogenesis, and was elevated to the maximal level 3-4 d after hatching. An assay of LRAT and ARAT activities along the villus-crypt axis of the duodenum by a cryostat sectioning technique revealed that between the day of hatching and $1 \mathrm{~d}$ posthatch, an abrupt induction of LRAT activity occurred only in the villus region of the duodenum, where a coordinated induction of cellular retinol-binding protein, type II (CRBPII), was observed. In contrast, the rise in ARAT activity observed around the hatching period occurred at the broader portions of the villi including the area of villus-crypt junction. These observations in the developmental changes and distribution of LRAT and ARAT activities suggest that LRAT activity but not ARAT activity is closely related to the induction of CRBPII in the duodenum of developing chicks.
\end{abstract}

Key Words lecithin-retinol acyltransferase (LRAT), acyl-CoA-retinol acyltransferase (ARAT), villus, small intestine, development

\footnotetext{
* To whom correspondence should be addressed.

E-mail: gouda@fns1.u-shizuoka-ken.ac.jp
} 
In vitamin A absorption in the small intestine, esterification of retinol is the final step which precedes its incorporation into chylomicrons and the export of absorbed vitamin A to lymph (1). The retinol uptaken into the enterocytes has been shown to bind to cellular retinol-binding protein, type two (CRBPII). This protein-ligand complex serves as the substrate for acyl-CoA independent retinol esterifying activity in the microsomes (2). This enzyme was named lecithin-retinol acyltransferase or phosphatidylcholine-retinol $O$-acyltransferase (LRAT, EC 2.3.1.135) since its activity transfers acyl moieties from the $\mathrm{C}-1$ position of phosphatidylcholine to retinol to produce retinyl esters (3). A second retinol esterifying activity has been described and designated as acyl-CoA-retinol acyltransferase or retinol $O$-fatty-acyltransferase (ARAT, EC 2.3.1.76) (4), which catalyzes esterification of free retinol, but not the retinol bound to CRBPII (2). We have previously demonstrated that the CRBPII in chick small intestine is rapidly induced around the hatching period (5), in parallel with the elevation of serum retinol concentration (6). This appearance of CRBPII in the developing chick intestine may lead to an increase in its capacity to absorb and transport retinol.

Ong et al (7) demonstrated, using immunohistochemical techniques, that rat CRBPII was present in the absorptive cells of the epithelium of the small intestine. We previously showed, using a cryostat slicing technique, that the initial induction of CRBPII in the chick small intestine occurred in differentiating lower villus enterocytes during the perinatal period, and that it was followed by a marked increase in the CRBPII content in the mid-villus cells (8). However, it has been unclear whether the retinol esterifying enzymes are also induced in the villus enterocytes during embryogenesis in the chick small intestine. Thus, questions arose as to whether the developmental changes and distribution of LRAT or ARAT activity correlated with those of CRBPII. To answer these questions, we examined the developmental changes of LRAT and ARAT activities in the duodenal mucosa as well as in cryostat-sectioned tissues at various heights of the villus of chick duodenum.

\section{MATERIALS AND METHODS}

Animals. Fertile eggs of the white leghorn breed were incubated at $38^{\circ} \mathrm{C}$ with $60 \%$ relative humidity. Hatched chicks were given free access to a commercial chick starter ration (Broiler-Diet, Tokai Kumiai, Aichi, Japan) and deionized water $1 \mathrm{~d}$ after hatching. The commercial diet was composed of $22 \%$ protein, $62 \%$ carbohydrate, $4 \%$ fat, $4 \%$ cellulose, $7 \%$ mineral and $1 \%$ vitamins. The diet contained approximately 2,650 IU vitamin A (as retinyl ester) per $100 \mathrm{~g}$ diet.

Duodenum was isolated from day 15, 18 and 20 embryos, and 0, 1, 3, 8, 14 and 22-d-old chicks.

Preparation of cryostat samples. To assay the enzyme activities at various heights of villus, a $5 \times 5 \mathrm{~mm}$ segment of duodenum was obtained from day 20 embryos and 0 and 1-d-old chicks, and it was sectioned with a cryostat at $-20^{\circ} \mathrm{C}$ 
as described previously (9). Horizontal sections were mounted on a microscope slide for examination of the villus and crypt architecture after staining with $0.3 \%$ methylene blue.

Preparation of microsomes for LRAT and ARAT assays. Microsomes were prepared from the homogenates as described previously (10). Briefly, the duodenum was homogenized in $2 \mathrm{vol}$. ( $\mathrm{v} / \mathrm{w})$ of ice-cold $0.2 \mathrm{M}$ potassium phosphate buffer $(\mathrm{pH}$ 7.2) using a Teflon-glass homogenizer. After centrifugation of homogenates at $10,000 \times g$ for $15 \mathrm{~min}$ at $4^{\circ} \mathrm{C}$, the resulting supernatant was followed by recentrifugation at $105,000 \times g$ for $1 \mathrm{~h}$ at $4^{\circ} \mathrm{C}$. The microsomal pellets were suspended in 1 vol. of $0.2 \mathrm{M}$ potassium phosphate buffer $(\mathrm{pH} 7.2)$ containing $1 \mathrm{~mm}$ dithiothreitol (DTT) and quickly frozen in liquid nitrogen before storage at $-80^{\circ} \mathrm{C}$.

The protein content of the microsome preparation was determined by the method of Lowry et al (11).

Assay of LRAT and ARAT. LRAT and ARAT activities were assayed with the conditions of protein concentrations and incubation times that had been validated for initial rate determinations. The LRAT activities were determined as described previously (10) according to the procedure of Ong et al (2) using retinol bound to CRBPII as the substrate. The CRBPII was purified from chick intestine (5) and the retinol-CRBPII complex was prepared as described previously (10).

The retinol-CRBPII $(3 \mu \mathrm{M})$ was incubated for $10 \mathrm{~min}$ at $37^{\circ} \mathrm{C}$ with the microsomes ( $60 \mu \mathrm{g}$ protein) in $0.2 \mathrm{~m}$ potassium phosphate buffer $(\mathrm{pH} 7.2)$ containing $2 \mathrm{~mm}$ DTT, $80 \mu \mathrm{m}$ dilauroyl phosphatidylcholine (Wako Pure Chemical Industries, Osaka, Japan) and $2.4 \mathrm{~g} / \mathrm{L}$ bovine serum albumin in a total volume of $250 \mu \mathrm{L}$. The reaction was terminated by adding $1 \mathrm{~mL}$ of ice-cold ethanol containing $100 \mathrm{mg} / \mathrm{L}$ butylated hydroxytoluene (BHT), and the produced retinyl esters were extracted from the reaction mixture into $4 \mathrm{~mL}$ hexane containing BHT $(100 \mathrm{mg} / \mathrm{L})$ as described previously (10). A portion of the hexane phase was evaporated to dryness under nitrogen gas and dissolved in $50 \mu \mathrm{L}$ methanol for high-performance liquid chromatography (HPLC) analysis. The HPLC was performed using a Shimadzu LC-6A system (Shimadzu Corporation, Kyoto, Japan) fitted with a $\mu$ Bondapak C18 column ( $10 \mathrm{~nm}$ particle, $3.9 \mathrm{~mm} \times 30 \mathrm{~cm}$, Water Associates, Milford, USA) with $100 \%$ methanol as the mobile phase at a flow rate of $2 \mathrm{~mL} / \mathrm{min}$. Absorbance of retinyl laurate in the eluates was determined using a spectrometer (SPD-6A, Shimadzu Corporation) with a wavelength of $330 \mathrm{~nm}$.

The ARAT activity was assayed according to a procedure described previously $(2,12)$. The reaction was initiated by incubating duodenal microsomes ( $30 \mu \mathrm{g}$ protein) with $30 \mu \mathrm{M}$ retinol (dispersed in dimethyl sulfoxide) and $80 \mu \mathrm{M}$ lauroyl-CoA in $0.2 \mathrm{M}$ potassium phosphate buffer ( $\mathrm{pH} 7.2$ ) containing $2.4 \mathrm{~g} / \mathrm{L}$ bovine serum albumin and $5 \mu \mathrm{M}$ DTT for $20 \mathrm{~min}$ at $37^{\circ} \mathrm{C}$. The total volume of the reaction mixture was $250 \mu \mathrm{L}$. The extraction of the reaction mixture and HPLC analysis of the extracts were performed by the same procedure described above for the LRAT assay.

The external standard of retinyl palmitate (Wako Pure Chemical Industries) was purified by HPLC, and its purity was more than $99 \%$ according to the HPLC 

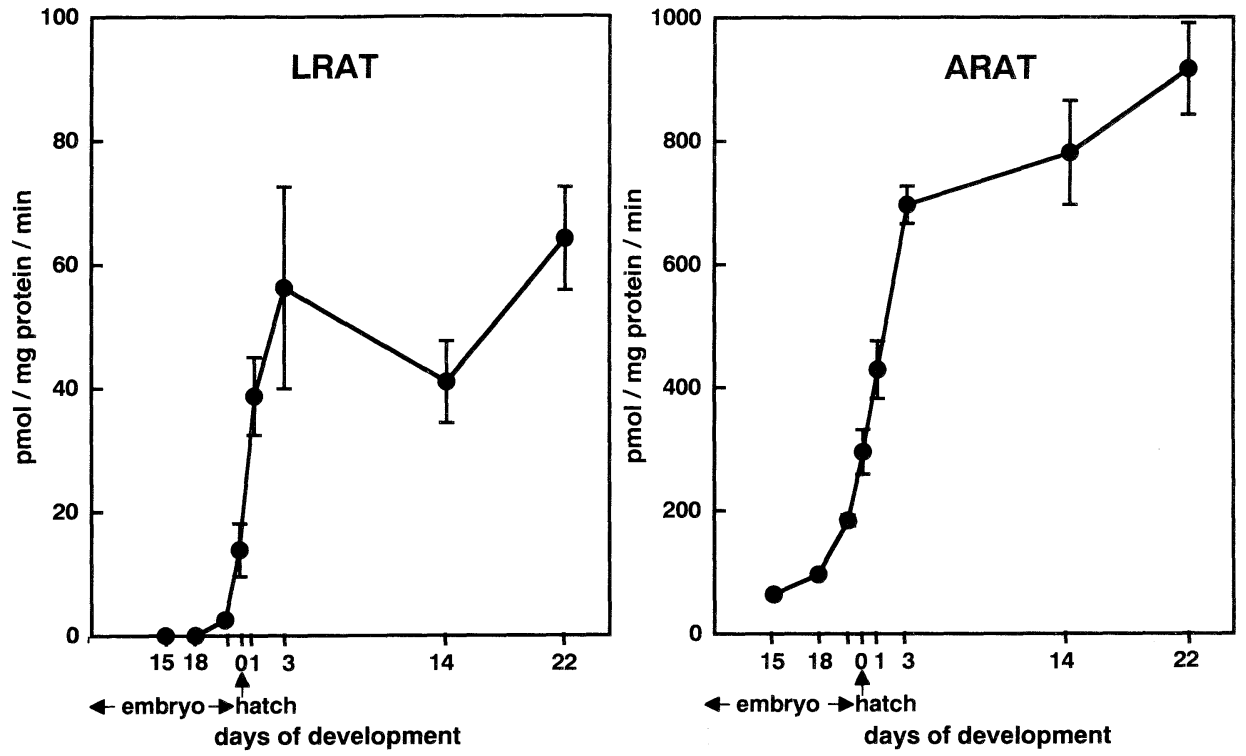

Fig. 1. Developmental changes of LRAT and ARAT activities in chick duodenum. The numbers prior to hatching refer to the days of incubation of chick embryos. The graphs show the means $\pm \mathrm{SE}$ for $3-4$ samples. The activity was expressed as $\mathrm{pmol}$ retinyl laurate-produced/mg microsomal protein $/ \mathrm{min}$.

profile.

RESULTS

Developmental changes in LRAT and ARAT activities in chick duodenum

Microsomes obtained from the small intestines of each developing stage were examined for LRAT and ARAT activities. The patterns of developmental changes in these enzyme activities are shown in Fig. 1. On days 15 and 18 of embryonic development, the duodenum exhibited very little LRAT activity. The LRAT activity slightly increased until day 20 of the embryonic stage, and during the subsequent $48 \mathrm{~h}$ (i.e., by day 1 after hatching) LRAT activity increased 15 times. A maximal level of LRAT activity $(56.2 \mathrm{pmol} / \mathrm{mg}$ protein $/ \mathrm{min})$ was observed on day 3 after hatching, and its level did not change thereafter. In contrast, considerable ARAT activity $(64.1 \mathrm{pmol} / \mathrm{mg}$ protein $/ \mathrm{min})$ was detected as early as day 15 of embryonic development. Thereafter, ARAT activity gradually increased reaching a maximal level at the age of $3 \mathrm{~d}(696.5 \mathrm{pmol} / \mathrm{mg}$ protein $/ \mathrm{min})$. This highest level was maintained throughout the posthatch period examined. 


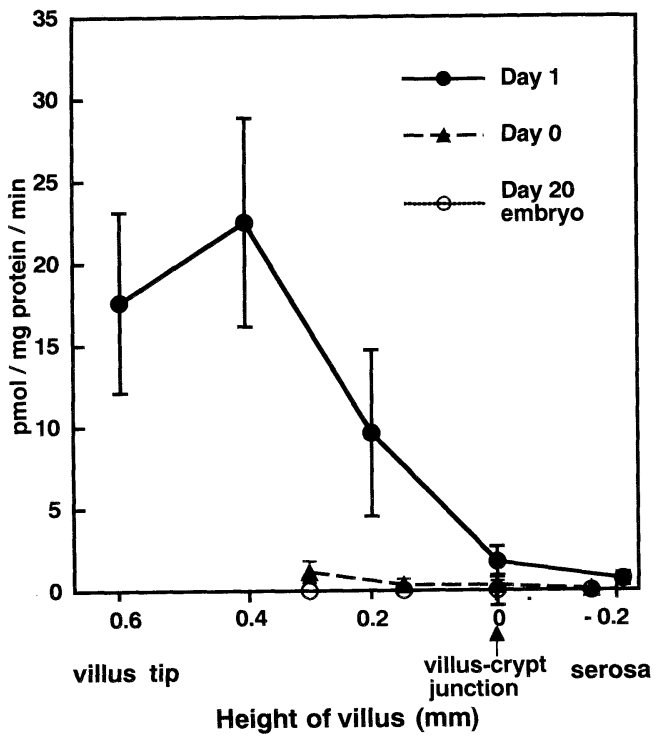

Fig. 2. Developmental changes in chick duodenal LRAT activity along the villus-crypt axis. Abscissa depicts the height of the villus in millimeters with zero (arrow) representing the villus-crypt junction. Means $\pm \mathrm{SE}$ are given for 3 chicks of each stage.

Developmental changes in duodenal LRAT and ARAT activities along the villus-crypt columns

To explore whether LRAT activity or ARAT activity is induced in the same region of enterocytes along the villus-crypt axis as CRBPII, we determined the developmental changes of LRAT and ARAT activities along the villus-crypt column of chick duodenum. As shown in Fig. 2, day 20 embryonic chicks did not exhibit any detectable LRAT activity along the villus-crypt columns of the duodenum. A slight increase in LRAT activity was observed in the villus regions on day 0 . During the following $24 \mathrm{~h}$, the villus length increased by approximately $100 \%$. This morphological change was accompanied by a marked increase in LRAT activity in the villus regions, demonstrating a typical villus-crypt gradient similar to that observed in 8-d-old chicks (13).

Figure 3 shows the developmental change in ARAT activity along the villus-crypt columns of the chick duodenum. On day 20 of embryonic development, the duodenum already exhibited a considerable level of ARAT activity which was evenly distributed along the villus-crypt column (Fig. 3). Immediately after hatching, the activity increased along the entire villus regions. In 1-d-old chicks, ARAT activity showed a characteristic distribution along the villus-crypt axis, in which the ARAT activity level was only slightly greater in the upper villus region than in the lower villus and crypt regions. The level of ARAT activity in the duodenal villus tip of 1-d-old chicks was 5 times greater than that in the villus tip of day 20 


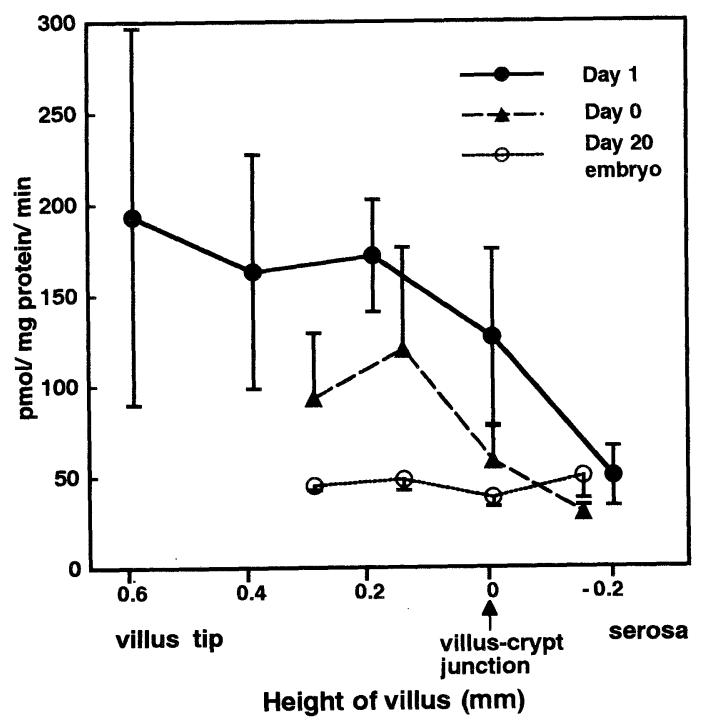

Fig. 3. Developmental changes in chick duodenal ARAT activity along the villus-crypt axis. Abscissa depicts the height of the villus in millimeters with zero (arrow) representing the villus-crypt junction. Means $\pm \mathrm{SE}$ are given for 3 chicks for each stage.

embryos (Fig. 3).

\section{DISCUSSION}

The retinol uptaken by enterocytes is possibly esterified by two distinct enzymes (i.e., LRAT (3) and ARAT (4)). We previously demonstrated that both CRBPII level and LRAT activity were very low in the small intestine of embryonic chicks, and they concomitantly and rapidly increased around the hatching period $(5,10)$. The abrupt increase in CRBPII protein content observed during the perinatal period was accompanied by a concomitant rise in CRBPII mRNA level (14). Therefore, it is most likely that CRBPII is induced at the genetic level during this critical period in the chick. This induction of CRBPII protein content in the small intestine of the chick was accompanied by a parallel increase in serum retinol concentration (6). This retinol was provided from the luminal contents derived from egg yolk that had penetrated the chick abdomen. In this perinatal period, chicks did not receive any food, yet retinol was detectable in their duodenal lumen. Therefore, it is possible that luminal vitamin A may play a role in the induction of intestinal CRBPII.

In this study, we investigated the developmental changes of LRAT and ARAT activities to seek the possibility that these two retinol esterifying enzymes play distinct roles in retinol absorption and metabolism in the small intestine. The LRAT activity in the small intestine was also extremely low in chick embryos, but LRAT 
activity rapidly rose around hatching (Fig. 1), in parallel with the induction of intestinal CRBPII (5). In contrast, ARAT activity was observed in the embryonic stage (Fig. 1). Therefore, it is possible that luminal vitamin A may also play a role in the induction of LRAT expression but not that of ARAT. On the other hand, we have also demonstrated that chicks fed a vitamin A-depleted diet showed a gradual increase in duodenal LRAT activity after $4 \mathrm{~d}$ of age (10), suggesting that putative genetic control is basically present and it may be dominant for LRAT induction in the perinatal period. These results led us to speculate that the perinatal development of LRAT activity and CRBPII is basically controlled by some common genetic programming and that the level of LRAT activity is further modulated by the vitamin A present in the lumen. Thus, it is likely that the increase in LRAT activity in this period is genetically determined by the maturation of enterocytes, being accelerated by the enhancement of retinol absorption.

We found that a prominent expression of LRAT activity was observed only after hatching and that an abrupt induction of LRAT activity occurred exclusively in the villus cells in the perinatal period (Fig. 2). The induction of LRAT observed in this period seems to be closely related with the abrupt increase in the expression of CRBPII, which is known to occur in the villus cells around the hatching period (8). This shows a clear contrast to the induction of ARAT activity, which has been induced before hatching (Fig. 3). For the vitamin A required in the development of embryonic tissues, retinol is provided from the yolk in embryonic chicks. During this embryonic stage, when neither CRBPII nor LRAT is expressed, ARAT may play a role in the absorption of retinol. It is most likely that the induction of CRBPII around the hatching period results in an exclusive binding of retinol to CRBPII, which would not be directed to ARAT. Therefore, it is considered physiologically significant that CRBPII and LRAT, but not ARAT, are coordinately expressed. We previously demonstrated that the distribution of the content of retinyl esters along the villus-crypt column of chick duodenum was similar to that of LRAT activity (13). These findings support the notion that, in the mature villus cells, LRAT is more physiologically related to retinol absorption and metabolism than ARAT. To examine our hypothesis that LRAT and CRBPII genes are coordinately regulated by common factor(s), it is inevitable to clone the chick LRAT gene and analyze the expression of the gene at the protein and mRNA levels as well as by transfection experiments in vitro. Recently, the LRAT was cloned from human retinal pigment epithelium and characterized at the molecular level (15). Further study requires the cloning of chick LRAT to elucidate the molecular mechanism of the regulation of LRAT gene expression, which possibly plays a pivotal role in vitamin A absorption and metabolism.

\section{REFERENCES}

1) Goodman DS, Blaner WS. 1984. Biosynthesis, absorption, and hepatic metabolism of retinol. In: The Retinoids (Sporn MB, Roberts AB, Goodman DS, eds), Vol 2, p 2-39. 
Academic Press, New York.

2) Ong DE, Kakkad B, MacDonald PN. 1987. Acyl-CoA independent esterification of retinol bound to cellular retinol-binding protein (type II) by microsomes from rat intestine. J Biol Chem 262: 2729-2736.

3) MacDonald PN, Ong DE. 1988. A lecithin: retinol acyltransferase activity in human and rat liver. J Biol Chem 263: 12478-12482.

4) Helgerud P, Petersen LB, Norum KR. 1982. Acyl CoA:retinol acyltransferase in rat small intestine: its activity and some properties of the enzymic reaction. $J$ Lipid Res 23: 609-618.

5) Goda T, Takase S. 1989. Purification, properties, and developmental changes of cellular retinol-binding protein, type II, in chicken intestine. J Nutr Sci Vitaminol 35: 545-557.

6) Takase S, Goda T. 1990. Developmental changes in vitamin A level and lack of retinyl palmitate in chick lung. Comp Biochem Physiol 96B: 415-419.

7) Ong DE, Lucus PC, Kakkad B, Quick TC. 1991. Ontogeny of two vitamin A-metabolizing enzymes and two retinol-binding proteins present in the small intestine of rat. J Lipid Res 32: 1521-1527.

8) Goda T, Pacifici M, Takase S. 1993. Induction and distribution of cellular retinol-binding protein, type two during villus-crypt development in the chick duodenum. Biol Neonate 64: 392-398.

9) Goda T, Yamada K, Bustamante S, Koldovský O. 1983. Dietary-induced rapid decrease of microvillar carbohydrase activity in rat jejunoileum. Am J Physiol 245: G418-G423.

10) Goda T, Furuta S, Takase S. 1993. Dietary vitamin A modulates lecithin: retinol acyltransferase activity in developing chick intestine. Biochim Biophys Acta 1168: $153-157$.

11) Lowry OH, Rosebrough NJ, Farr AL, Randall RJ. 1951. Protein measurement with the Folin phenol reagent. $J$ Biol Chem 193: 265-275.

12) Randolph RK, Ross AC. 1991. Vitamin A status regulates hepatic lecithin: retinol acyltransferase activity in rats. $J$ Biol Chem 266: 16453-16457.

13) Tajima S, Goda T, Takase S. 1999. Coordinated distribution patterns of three enzyme activities involved in the absorption and metabolism of $\beta$-carotene and vitamin $\mathrm{A}$ along the villus-crypt axis of chick duodenum. Life Sci 65: 841-848.

14) Suruga K, Goda T, Igarashi M, Kato S, Masushige S, Takase S. 1997. Cloning of chick cellular retinol-binding protein, type II and comparison to that of some mammals: Expression of the gene at different developmental stages, and possible involvement of RXRs and PPAR. Comp Biochem Physiol 118A: 859-869.

15) Ruiz A, Winston A, Lim Y-H, Gilbert BA, Rando RR. 1999. Molecular and biochemical characterization of lecithin : retinol acyltransferase. J Biol Chem 274: 3834-3841. 